\title{
Additive Value of Transrectal Systematic Ventral Biopsies in Combination with Magnet Resonance Imaging/Ultrasound Fusion-Guided Biopsy in Patients with 3 or More Negative Prostate Biopsies
}

\author{
Andreas Maxeiner ${ }^{a} \quad$ Alexander M. Nest $^{a} \quad$ Carsten Stephan $^{a}{ }^{b} \quad$ Hannes Cash $^{a}$ \\ Alexander D.J. Baur ${ }^{c}$ Thomas Fischer ${ }^{c}$ Ergin Kilic ${ }^{d, e}$ Sophie K. Piper ${ }^{f}$ \\ Claus-P. Nowak ${ }^{f}$ Jonas Busch ${ }^{a} \quad$ Kurt Miller $^{\mathrm{a}} \quad$ Josef Mang $^{\mathrm{a}}$ \\ ${ }^{a}$ Charité, Universitätsmedizin Berlin, Corporate Member of Freie Universität Berlin, Humboldt-Universität zu Berlin,

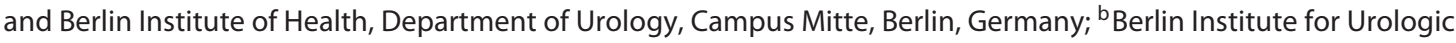 \\ Research, Berlin, Germany; ' $C$ arité, Universitätsmedizin Berlin, Corporate Member of Freie Universität Berlin, \\ Humboldt-Universität zu Berlin, and Berlin Institute of Health, Department of Radiology, Campus Mitte, Berlin, \\ Germany; ${ }^{\mathrm{d}}$ Charité, Universitätsmedizin Berlin, Corporate Member of Freie Universität Berlin, Humboldt-Universität \\ zu Berlin, and Berlin Institute of Health, Department of Pathology, Campus Mitte, Berlin, Germany; ${ }^{\mathrm{e}}$ Institute of \\ Pathology, Klinikum Leverkusen, Leverkusen, Germany; ${ }^{f}$ Charité, Universitätsmedizin Berlin, Corporate Member \\ of Freie Universität Berlin, Humboldt-Universität zu Berlin, and Berlin Institute of Health, Institute of Biometry and \\ Clinical Epidemiology, Campus Mitte, Berlin, Germany
}

\section{Keywords}

Prostate cancer · Multiparametric magnet resonance imaging · Fusion-guided biopsy · Systematic TRUS-guided biopsy · Repeat biopsy

\begin{abstract}
Introduction: Patients with consistent suspicion for prostate cancer (PCa) and multiple negative prebiopsies prior to multiparametric magnetic resonance imaging (mpMRI) are still frequently evaluated for an image-guided biopsy and are reported with heterogeneous detection rates. The inclusion of a systematic biopsy (SB) is also still recommended with predominant sampling within the posterior/peripheral zone of the prostate. The aim of this study was (I) to evaluate PCa detection rates using a modified 10 core SB template includ-
\end{abstract}

karger@karger.com

(c) 2019 S. Karger AG, Basel

www.karger.com/uin

Karger ing anterior biopsies in combination with $\mathrm{mpMRI} / \mathrm{ultra}$ sound fusion-guided targeted biopsy (TB) in patients with 3 or more negative prebiopsies and (II) to compare mpMRI index lesion localization with histologically confirmed localization from associated prostatectomy samples. Methods: Overall 1,337 consecutive patients underwent sensor-based registration TB of the prostate and a subsequent 10-core SB between January 2012 and December 2015 at our institution. For this study, 101 patients with $\geq 3$ negative prebiopsies and prostate imaging - reporting data system lesions $\geq 3$ were pooled prospectively and underwent TB and a modified SB including 2 ventral (anterior) biopsies. Detection rates were estimated for the modified $\mathrm{SB}, \mathrm{TB}$, and its combi-

A.M. and A.M.N. contributed equally to this work. 
nation. A subgroup analysis of 35 patients undergoing prostatectomy was performed by a head-to-head comparison of $\mathrm{mpMRI}$ index lesion and histologically confirmed PCa index lesion localization. Results: The overall detection rate for PCa was $54.5 \%$. The combination of TB and SB detected 14 (25.4\%) more cases missed by TB alone $(p<0.001)$ and 7 (12.7\%) more cases missed by SB alone ( $p=0.016)$, respectively. A postoperative Gleason upgrade was seen in 12/35 (34.3\%) cases within the TB group and in 14/35 (40.0\%) in the SB group, respectively. The subgroup analysis showed a predominant location of PCa index lesions anteriorly at the level of the midgland. The MRI detection rate of the anteriorly located index lesions was $70.4 \%$ ( $15 / 21$ cases) with a clinically significant Gleason score $(\geq 3+4=7$ a [International Society of Urological Pathology grade 2]) in $80.9 \%$. Interestingly a modified SB template detected $90.5 \%(19 / 21)$ of the anteriorly located index lesions. Conclusion: Our data suggest that in patients with multiple prebiopsies PCa seems to be predominantly located anteriorly. We suggest the general integration of anterior biopsies despite TB in repeat biopsy patients.

(C) 2019 S. Karger AG, Basel

\section{Introduction}

Implementation of multiparametric magnet resonance imaging (mpMRI) in the prostate cancer $(\mathrm{PCa}) \mathrm{di}-$ agnostic pathway [1] and the application of MRI/ultrasound (US) fusion targeted biopsy (TB) have resulted in increased PCa detection rates [2]. The aim of mpMRI is to identify $\mathrm{PCa}$ index lesions representing the largest tumor focus and also clinically significant PCa (usually associated with the highest Gleason score) by using a standardized prostate reporting system (the prostate imaging - reporting data system [PI-RADS]) [3-5]. The combination of mpMRI and TB plus a subsequent systematic biopsy (SB) has high detection rates of clinically significant PCa [6]. While mpMRI already shows high sensitivities for detecting clinically significant PCa index lesions [7], the combination of TB and subsequent SB seems to be superior in detecting significant PCa to mpMRI alone $[8,9]$. Especially small nonindex lesions representing clinically significant $\mathrm{PCa}$ can be missed by mpMRI and TB alone [10].

However, in biopsy-naïve men, the recently published PRECISION trial showed that MRI could be used as prebiopsy risk assessment if MRI results were not suggestive of PCa [11]. Furthermore, the PROMIS study concluded in case of mpMRI as a triage test before first prostate bi- opsy showed that MRI could identify a quarter of men who might safely avoid an unnecessary biopsy and might improve the detection of clinically significant cancer [12]. In addition, a recently published high-quality standard study showed that in biopsy-naïve men undergoing mpMRI, biopsy may be omitted in half of men, and fewer insignificant $\mathrm{PCa}$ are detected by only missing $4 \%$ clinical significant PCa by not performing SB [13].

But during repeat biopsy, the inclusion of a 10-12 core SB in combination with a TB is still recommended [14].

Despite the promising results of using $\mathrm{mpMRI}$ in $\mathrm{PCa}$ diagnostics, still patients with multiple prebiopsies prior to TB can be observed. Detection rates of SB at initial biopsy of $20-30 \%$ with constant decrease down to $4-12 \%$ at the time of a 4 th biopsy have been reported $[15,16]$. Concerning the histopathological localization of PCa in biopsy-naïve patients, it seems to be predominantly located with up to $90 \%$ within the posterior part of the prostate, whereas only $10 \%$ is located anteriorly $[17,18]$. In contrast, PCa localization differs drastically in patients with prior negative biopsies, where PCa is located in the anterior part of the prostate in about $50-80 \%$ [19-21]. This is reflected by modified protocols for SB in a rebiopsy setting, such as including the anterior prostate region in the biopsy protocol and increasing the number of cores taken [22].

However, there are little data available on histological findings, especially in patients with a history of at least 3 prior negative biopsies undergoing MRI/US fusion-guided biopsy. Hence, available information during MR imaging and potential histological data postoperatively need to be analyzed for that subgroup. To our knowledge combining TB with a modified 10 core SB including a left and right ventral (anterior) instead of a left and right lateral biopsy has not been reported yet. Therefore, the aim of this study was (I) to estimate TB, SB, and combined detection rates including a modified 10 core template in patients with at least 3 negative prebiopsies and (II) to compare MR index locations with postoperatively confirmed histological data.

\section{Methods}

Patient Population

A total of 1,337 consecutive patients underwent TB of the prostate and a subsequent 10-core SB between January 2012 and December 2015 at our institution. At the time of biopsy, all enrolled patients signed a written informed consent for the intervention, data acquisition, data appraisal, and publication according to the Declaration of Helsinki and authorized by the institutional ethical review board (EA1/283/14 and EA1/012/12). 
Fig. 1. Histopathological correlation of MRI prelocated index lesions location according to PI-RADS: (a) PI-RADS template for standardized reporting (a, anterior; p, posterior; 25); (b) representative DWI axial section with ADC-map of an anteriorly and centrally located index lesion within the midgland level with a $5 \mathrm{~mm}$ grid overlay; (c) example of a histopathological hematoxylin-eosin (HE) slice with marked malignant area of a matching lesion anteriorly and centrally located at the midgland level with a $5 \mathrm{~mm}$ grid overlay; (d) the representing anterior quarter within the PIRADS template at the midglandlevel.

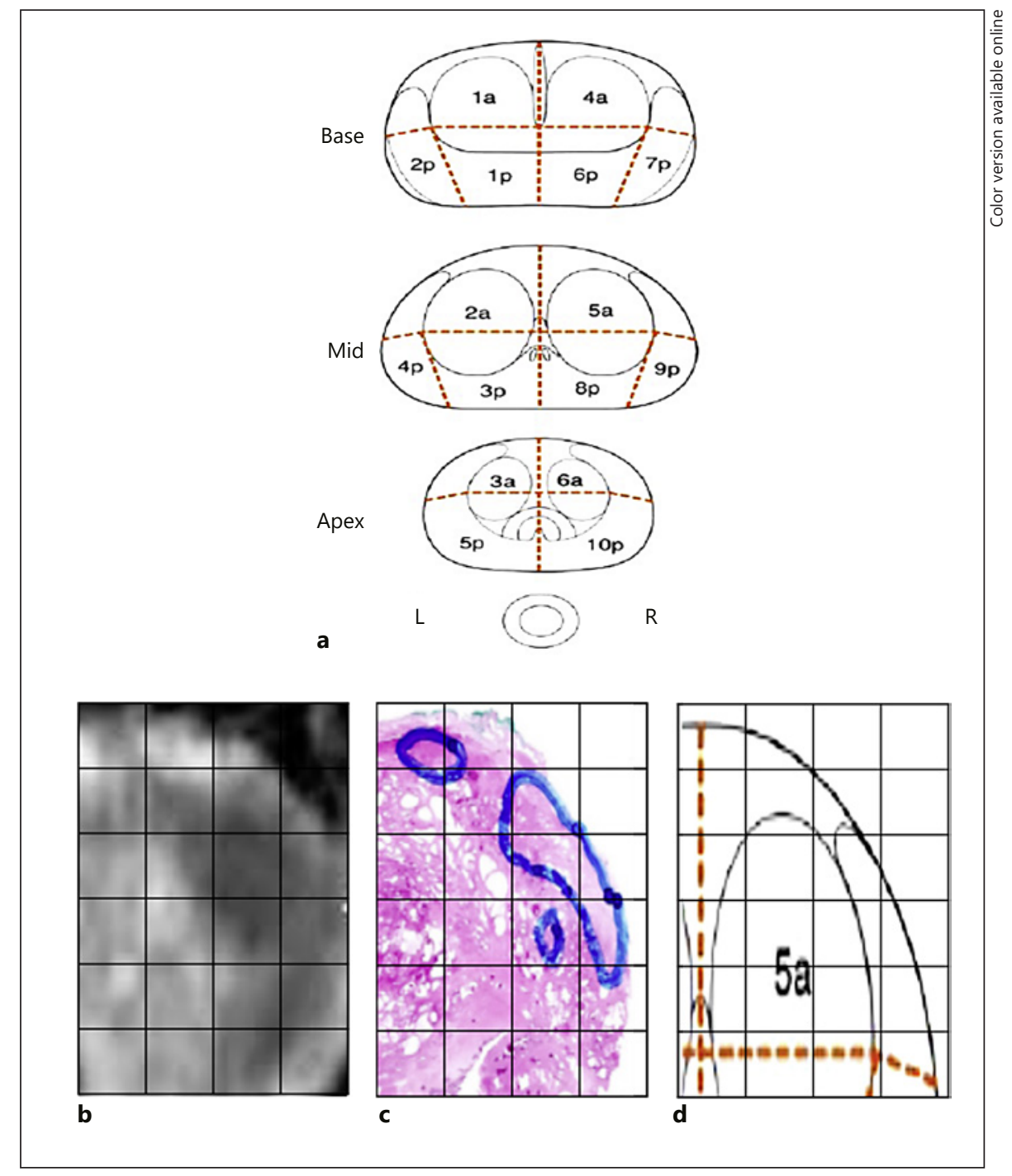

For the study at hand, all patients with a normal DRE and a history of at least 3 standard systematic 10-12-core transrectal ultrasound (TRUS) biopsies with no histological proof of PCa or highgrade prostatic intraepithelial neoplasia underwent a modified 10core $\mathrm{SB}$ subsequent to $\mathrm{TB}$ including 2 ventral (anterior) biopsies. Based on these inclusion criteria, we were able to pool 108 patients prospectively. We had to exclude 3 patients based on mpMRI acquisition artefacts, 2 patients who had refused a complete SB, and 2 patients who had been operated in different hospitals and for whom insufficient postoperative histopathological data were available. Thus, 101 patients finally met study inclusion criteria.

\section{Multiparametric Magnetic Resonance Imaging}

All included patients underwent a 3 Tesla mpMRI (Magnetom Skyra, Siemens Medical Systems, Erlangen, Germany) without endorectal coil using standardized imaging protocols in accordance with current guidelines. This protocol included multiplanar (axial and coronal), high-spatial resolution T2-weighted turbo spinecho sequences (T2w TSE), axial T1-weighted images (T1w), axial diffusion weighted images ([DWI] measured b values 0,400 , and $800 / \mathrm{mm}^{2}$, calculated b value of $1,000-1,400 \mathrm{~s} / \mathrm{mm}^{2}$ ) and dynamic contrast-enhanced sequences (DCE-MRI). T2-weighted imaging and DWI were performed in all patients. DCE-MRI was only performed in only $77(76.2 \%)$ patients. The evaluation and validation of the mpMRI image data were performed or supervised by experienced uro-radiologists in compliance with the guidelines of the European Society of Urogenital Radiology according to PI-RADS version 1.0. The lesion with the highest PI-RADS score was defined as the index lesion in patients with multiple lesions and further included in the analysis. The location of the index lesion was described by 3 definitions: apical vs. mid gland vs. basal, peripheral vs. central, and ventral (anterior) vs. dorsal (posterior).

\section{MRI Fusion-Guided and Modified SB}

All included patient with a PI-RADS score of $\geq 3$ underwent TB and subsequent SB. Prior to the biopsy, antibiotic prophylaxis with a fluoroquinolone (i.e., Ciprofloxacin) was initiated. After image fusion by sensor-based registration, a TB with a median of 2 cores 
Table 1. Patient Characteristics $(n=101)$

\begin{tabular}{lc}
\hline Characteristics & \\
Age, years & $71(51-82)$ \\
PSA, ng/mL & $12.3(1.58-39)$ \\
Prostate volume, mL & $52(12-174)$ \\
PI-RADS score & $4(3-5)$ \\
Biopsies before TB, $n$ & $3(3-12)$ \\
Diagnosis & \\
PCa & $55(54.5)$ \\
BPH & $14(13.9)$ \\
Prostatitis & $6(5.9)$ \\
hgPIN & $2(1.9)$ \\
Normal tissue & $24(23.8)$ \\
Treatment of PCa $(n=55)$ & \\
oRP & $21(38.2)$ \\
RARP & $14(25.5)$ \\
AS & $12(21.8)$ \\
Radiation & $7(12.7)$ \\
Androgen deprivation therapy & $1(1.80)$ \\
cT-Stage $(n=55)$ & \\
cT1c & $48(87.3)$ \\
cT2a & $5(9.10)$ \\
cT2c & $2(3.60)$ \\
pT-Stage $(n=35)$ & \\
pT2a & $3(8.57)$ \\
pT2c & $21(60.0)$ \\
pT3a & $8(22.9)$ \\
pT3b & $2(5.70)$ \\
pT4 & $1(2.83)$ \\
\hline
\end{tabular}

Values are presented as $n(\%)$ or mean \pm SD. TB, target biopsy; $\mathrm{PCa}$, prostate cancer; $\mathrm{BPH}$, benign prostatic hyperplasia; hgPIN, high-grade intraprostatic neoplasia; oRP, open retropubic radical prostatectomy; RARP, robotic assisted radical prostatectomy; AS, active surveillance; ADT, androgen deprivation therapy; $\mathrm{CT}$, clinical stage; $\mathrm{pT}$, pathological tumor stage.

(range 2-4) of the prostate was performed, using the high-end US machine HiVison Preirus (Hitachi Medical Systems, Tokyo, Japan) or Aplio 500 (Toshiba [Canon Medical], Otawara, Japan) with an endocavity endfire probe (11C3, Toshiba [Canon Medical], Otawara, Japan; EUP V53 W, Hitachi medical Systems, Tokyo, Japan) or bipolar probe (EUP CC531, Hitachi, Medical Systems, Tokyo, Japan), as described previously [4]. Consecutively a modified 10 core systematic TRUS biopsy similar to a described apical anterior horn biopsy [23] without local anesthesia was performed. The template was consisting of right and left apical, right and left intermediate, right and left basal, right and left para-urethral, right and left ventral (anterior) biopsies. Concerning the right and left ventral (anterior) biopsy, the endorectal US probe was positioned in the sagittal plane, such that the needle guide was positioned anteriorly with respect to the probe. This modified template is performed in our institution since December 2012. Both $\mathrm{TB}$ and SB were performed by the same physicians (A.M., C.S., H.C.) in one biopsy session. All scores were plotted and documented separately.

\section{Histopathology and Correlation of Localization}

All biopsy specimens were examined and analyzed by an experienced uro-pathologist. After paraffin embedding and slicing, a hematoxylin-eosin (HE) staining was performed. A significant PCa was defined by GS $\geq 3+4=7$ (International Society of Urological Pathology [ISUP] grade 2). The overall total Gleason score of each patient was defined as the highest Gleason score of all scores. In all cases in which a prostatectomy was performed, the prostatectomy specimens were assessed according to the pathologic assessment of radical prostatectomy specimens (TNM 2009, WHO 2004, ISUP 2005): After fixation, the specimen was dried and painted with different colors using ink to orient the gland and to clearly delineate the surgical margins. Three-millimeter wholemount sections were divided into halves (or quadrants, in a large gland) to fit into standard-size cassettes used for paraffin embedding. Standard HE stains were performed on all sections, all apical and bladder neck sections and the single embedded sections from each seminal vesicle [24]. To perform also an estimation of tumor extent within the prostate gland, $\mathrm{PCa}$ lesions are manually circled on the HE stains. The location of the highest Gleason score was taken as the histopathological index tumor. To improve the comparability between histopathological sections and MR images, the microscopically identified index lesion was compared to a corresponding DWI with a $5 \mathrm{~mm}$ grid overlay. The location was similarly described to PI-RADS as apical vs. mid gland vs. basal, peripheral vs. central, and anterior vs. posterior (Fig. 1a-d) [25].

\section{Statistical Analysis}

All statistical calculations were performed using SPSS version 20 (IBM Corp., Somers, NY, USA). Results are reported as frequencies and percentages for all categorical outcomes. McNemar test was applied to test for asymmetries in detection frequencies between SD or TB alone versus SB and TB combined. Statistical tests were two-sided unless stated otherwise and a $p$ value $<0.05$ was taken to indicate statistical significance. No adjustment for multiple comparisons has been made, and all $p$ values constitute exploratory data analysis and do not allow for confirmatory generalization of results.

\section{Results}

Descriptive data are shown in Table 1 . The median age was 71 years (range 51-82 years), and the median PSA was $12.3 \mathrm{ng} / \mathrm{mL}$ (range $1.58-39 \mathrm{ng} / \mathrm{mL}$ ). Out of $101 \mathrm{in}$ cluded patients, the overall PCa detection rate was $54.5 \%$. The detected Gleason scores ranged from $3+3=6$ (ISUP1) in $25(45.5 \%)$ cases, $3+4=7 \mathrm{a}$ (ISUP2) in 8 (14.6\%) cases, $4+3=7 \mathrm{~b}$ (ISUP3) in 7 (12.7\%) cases, $4+$ $4=8$ (ISUP 4$)$ in $11(20.0 \%)$ cases, and $4+5=9$ (ISUP 5) in $4(7.20 \%)$ cases.

As shown in Table 2, TB detected 41 (74.5\%) PCas with the following distribution: $3+3=6$ (ISUP1) in $16(64.0 \%)$ cases, $3+4=7 \mathrm{a}$ (ISUP2) in $5(62.5 \%)$ cases, $4+3=7 \mathrm{~b}$ (ISUP3) in $7(100 \%)$ cases, $4+4=8$ (ISUP 4$)$ in $9(81.8 \%)$ cases, and $4+5=9$ or higher (ISUP 5 ) in 4 cases $(100 \%)$. 
Table 2. Comparison of positive biopsy depending on Gleason score

\begin{tabular}{lllllll}
\hline $\begin{array}{l}\text { Gleason } \\
\text { score }\end{array}$ & $\begin{array}{l}3+3=6 \\
(\text { ISUP 1) }\end{array}$ & $\begin{array}{l}3+4=7 \mathrm{a} \\
(\text { ISUP 2) }\end{array}$ & $\begin{array}{l}4+3=7 \mathrm{~b} \\
(\text { ISUP 3) }\end{array}$ & $\begin{array}{l}\geq 4+4=8 \\
(\geq \text { ISUP 4) }\end{array}$ & Total & $p$ value \\
\hline $\mathrm{SB}+\mathrm{TB}$ & 25 & 8 & 7 & 15 & 55 & - \\
$\mathrm{SB}$ & 20 & 8 & 7 & 13 & 48 & $0.016^{*}$ \\
$\mathrm{~TB}$ & 16 & 5 & 7 & 13 & 41 & $0.001^{*}$ \\
\hline
\end{tabular}

* McNemar's test comparing SB or TB alone with the combination of SB and TB. ISUP, International Society of Urological Pathology; SB, systematic biopsy; TB, target biopsy.

Table 3. Accordance of MRI and histopathological location and biopsy distribution

\begin{tabular}{|c|c|c|c|c|c|}
\hline & $\begin{array}{l}\text { MRI index } \\
\text { location }\end{array}$ & $\begin{array}{l}\text { Histological } \\
\text { index location }\end{array}$ & $\begin{array}{l}\text { True positive: } \\
\text { MRI matching } \\
\text { histological location }\end{array}$ & $\begin{array}{l}\text { Location of } \\
\text { positive TB }\end{array}$ & $\begin{array}{l}\text { Location of } \\
\text { positive SB }\end{array}$ \\
\hline Base & 7 & 1 & $1(100)$ & 1 & 1 \\
\hline Midgland & 23 & 26 & $20(76.9)$ & 19 & 22 \\
\hline Apex & 5 & 8 & $5(62.5)$ & 7 & 8 \\
\hline Total & 35 & 35 & $26(74.2)$ & 27 & 31 \\
\hline Posterior (dorsal) & 17 & 14 & $11(78.6)$ & 11 & 13 \\
\hline Anterior (ventral) & 18 & 21 & $15(71.4)$ & 16 & 19 \\
\hline Total & 35 & 35 & $26(74.3)$ & 27 & 32 \\
\hline Peripheral & 20 & 26 & $17(65.3)$ & 18 & 24 \\
\hline Central & 15 & 9 & $6(66.7)$ & 9 & 7 \\
\hline Total & 35 & 35 & $23(65.7)$ & 27 & 31 \\
\hline
\end{tabular}

MRI, magnetic resonance imaging; TB, target biopsy; SB, systematic biopsy.

In contrast, 48 (87.2\%) PCas have been detected by SB with the following distribution: $3+3=6$ (ISUP1) in 20 $(80 \%)$ cases, $3+4=7$ a (ISUP2) in $8(100 \%)$ cases, $4+3=$ $7 \mathrm{~b}$ (ISUP3) in $7(100 \%)$ cases, $4+4=8$ (ISUP 4 ) in 9 $(86.6 \%)$ cases, and $4+5=9$ or greater (ISUP5) in $4(100 \%)$ cases (Table 2). The combination of TB and SB detected $14(25.4 \%)$ additional cases that were missed by TB alone $(p<0.001 ;$ McNemar's test) and 7 (12.7\%) cases missed by SB alone ( $p=0.016$; McNemar's test). SB alone detected 7 more cases than TB alone, though did not reach statistical significance ( $p=0.189$; McNemar's test).

Thirty-five out of 55 PCa patients subsequently underwent radical prostatectomy. The postoperative Gleason score distribution was within this subgroup: $3+3=$ 6 (ISUP1) in $4(11 \%)$ cases, $3+4=7 \mathrm{a}$ (ISUP2) in 13 (37\%) cases, $4+3=7 \mathrm{~b}$ (ISUP3) in $11(31 \%)$ cases, $4+$ $4=8$ (ISUP 4$)$ in $2(6 \%)$ cases, and $4+5=9$ or $5+4=$ 9 (ISUP5) in 5 cases (15\%). The remaining other 20
(36.3\%) PCa cases underwent other treatments; displayed in Table 1.

A Gleason upgrade from biopsy results to postoperative Gleason scores was seen in 12/35 (34.3\%) cases within the TB group and in $14 / 35(40.0 \%)$ in the SB group, respectively.

The subgroup analysis showed based on histopathology a predominant location of the PCa index lesions anteriorly (21/35). The MRI detection rate of the anteriorly located index lesions was $70.4 \%$ (15/21 cases) with a clinically significant Gleason score $(\geq 3+4=7 \mathrm{a}$ [ISUP2]) in $80.9 \%$ of the cases. The modified SB template detected $90.5 \%(19 / 21)$ of the anteriorly located index lesions.

A head-to-head comparison of mpMRI and histopathology locations including the distribution of Gleason scores was plotted in Figure $2 \mathrm{a}$ and $\mathrm{b}$ and also distributed in Table 3. The visual distribution of the mpMRI loca- 
Fig. 2. 3D distribution of (a) MRI indexlesion location and associated Gleason score (ISUP; b) Histopathology index-lesion location and associated Gleason score (ISUP). Numbers represent individual patient IDs to allow for comparison of lesion localization. ISUP, International Society of Urological Pathology; PZ, peripheral zone; $\mathrm{CZ}$, central zone.
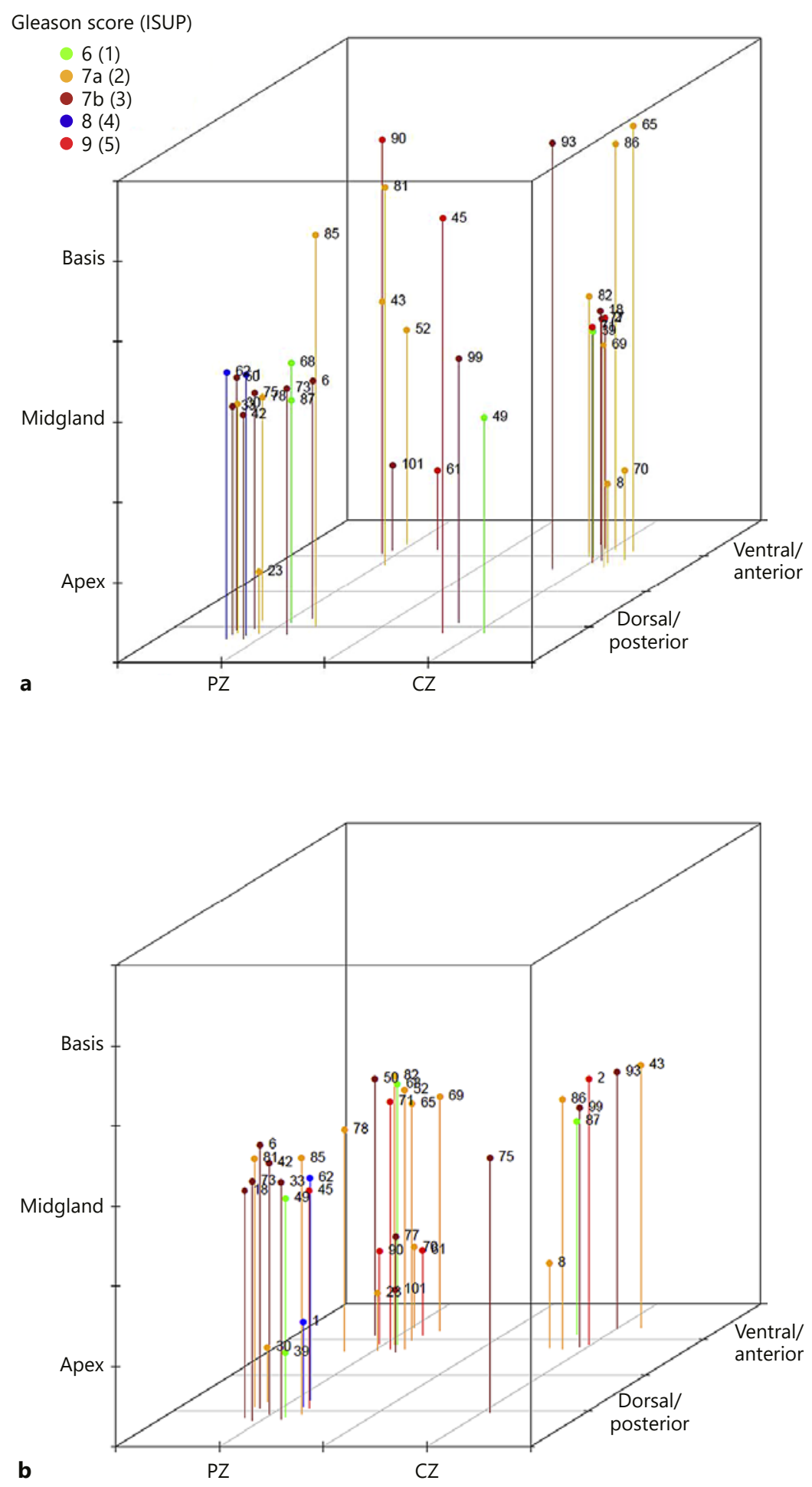

b

PZ

Maxeiner et al. 
tions compared to the histopathologically confirmed distribution in a heat map can be found within the online supplementary material (or all online suppl. material, see www.karger.com/doi/10.1159/000504266).

\section{Discussion}

There are studies reporting a limited additional value of SB in addition to TB $[2,22]$, but other authors also conclude that TB should be combined with SB for improved detection of clinically significant PCa [26-28]. However, the integration of MRI/US fusion into the diagnostic pathway in patients with suspicion for PCa with previous negative prostate biopsy led to an increased diagnostic accuracy for the detection of clinically significant PCa (Gleason score $\geq 3+4=7 \mathrm{a}$ (ISUP 2). Patients with suspicion for $\mathrm{PCa}$ and multiple negative prebiopsies are reported with heterogeneous detection rates in a random biopsy setting $[15,16,29]$. Extending the number of cores and locations may increase the diagnostic yield of $\mathrm{PCa}$, but it may also inappropriately increase the detection of insignificant $\mathrm{PCa}$, potentially leading to unnecessary treatments [11]. Conventional SB predominantly misses a potential proportion of PCa lesions located in the anterior segment of the prostate especially in patients with a reported increased number of anteriorly located PCa foci [30]. The results of our study with histologically $60 \%$ of pathologically confirmed anterior PCas strongly confirmed those data [25]. Therefore, we hypothesized that a modified standard 10 core template including 2 ventral (anterior) biopsies in addition to MRI/ US fusion-guided biopsy in patients with $\geq 3$ negative prebiopsies of the prostate would be beneficial for PCa detection.

Due to the results of the PRECISION trial [11], or the PROMIS group [12], or a large multicenter study of van der Leest et al. [13] a trend toward target biopsy as a primary approach for PCa detection can be observed. Patients with multiple negative prebiopsies before MRI might be rare in future. But according to the EAU guidelines [14], MRI is indicated in a repeat biopsy setting and $\mathrm{SB}$ is still indicated in addition to a TB. There are also little data about patients with multiple prebiopsies before TB. Our data seem to confirm that PCa lesions within these patients might predominantly be located anteriorly in both the MR images and also in the final histopathological samples. Based on our head-to-head comparison of histopathology and the associated imaging report after prostatectomy, still lesions have been either missed

Systematic Ventral Biopsies During MRI/

US Fusion-Guided Prostate Biopsy by the MRI in $28.6 \%$ (6/21 cases) or rated false positively in $16.6 \%$ ( $3 / 18$ cases). In contrast, the modified SB detected $90.5 \%$ of the PCa within in the anteriorly located lesions. The combination of the modified SB and TB detected both more clinically significant PCa and 100\% [21/21] of the histopathologically classified anterior lesions.

A recently published study of Schouten et al. [31] reports high rates of missed $\mathrm{PCa}$ lesions in biopsy-naïve men by SB especially involving segments located anteriorly. The study also concludes to obtain additional samples from the anterior apex and anterior midgland during $\mathrm{SB}$ as a consequence of their findings. Hence, knowledge of lesions being missed with TB and SB is of clinical importance for diagnosis and treatment and needs further investigation. Our results demonstrate the importance of anterior biopsies during SB in pre-biopsied patients. These findings are also supported by the fact of a significant Gleason upgrade in the final pathology of anterior lesions despite biopsy modalities. However, adding SB to TB detected 5 more patients with significant PCa but also 9 insignificant $\mathrm{PCa}$, potentially leading to overtreatment if active surveillance is not reasonably assessed.

Despite a positive role for the modified SB in PCa detection, we acknowledge further limitations to the present study. The study represents a single-center analysis without randomization, whereas multicenter randomized trials are desirable. Furthermore, the data set represents a highly selective patient cohort. Due to the awareness of the mpMRI, the urologist performing the SB might have resampled the respective areas during SB. Patients with a larger prostate volume might have been undersampled by a 10 core template [32]. Only patients with positive mpMRI (PI-RADS $\geq 3$ ) findings have been included and mpMRI was lacking of DCE in 30 cases but according to PI-RADS version 2.0, DCE seems to be beneficial only in PI-RADS 3 lesions. Finally, we performed the biopsy transrectally, whereas patients with anteriorly located lesions could benefit from a perineal approach $[33,34]$. Especially MRI-targeted perineal biopsy with in a repeat setting seems to increase the detection of PCa within the anterior zone [35].

Based on our study in hand, the combination of SB and TB seems to detect both more and clinically significant PCa. Furthermore, our cohort includes radical prostatectomy as the reference standard in most patients at least in our subgroup analysis and represents a good and strong association with an anterior localization of PCa foci in patients with multiple prebiopsies before imaging and consecutive TB. 


\section{Conclusion}

Our study demonstrates a cumulative appearance of anterior PCa lesions in both mpMRI of the prostate and final histopathology in patients with multiple prebiopsies before $\mathrm{TB}$ and subsequent radical prostatectomy. The number of clinically significant cancer seems also be increased in these patients and especially within the anterior region. Mp-MRI is strongly indicated in patients referred for a repeat biopsy. Although associated with a significant proportion of Gleason upgrade in the final pathology, adding a modified systematic template biopsy with 2 additive ventral (anterior) biopsies seems to be beneficial to these patients.

\section{Acknowledgments}

We cordially thank Gunilla Maxeiner and Klaus Jung for carefully reading the manuscript. We would also like to thank Gertrud Feldman for her excellent work at the outpatient clinic.

\section{Statement of Ethics}

All enrolled patients signed a written informed consent for the intervention, data acquisition, data appraisal, and publication according to the Declaration of Helsinki and authorized by the institutional ethical review board of the Charite - Universitätsmedizin Berlin: EA1/283/14 and EA1/012/12.

\section{Disclosure Statement}

T.F. reports receiving honoraria as a speaker on national and international conferences for Siemens, Canon medical (Toshiba) and Hitachi, CEUS Advisory Board Member for Bracco. H.C. reports receiving honoraria as a speaker on national and international conferences for Hitachi. A.M. reports receiving honoraria as a speaker on national and international conferences for Canon medical (Toshiba). A.D.J.B. has received payments as a speaker for Bayer Pharma AG and b.e.imaging $\mathrm{GmbH}$.

\section{Funding Sources}

There is no specific funding related to the subject matter of the article.

\section{Author Contributions}

A.M. and J.M.: designed the research study. A.M., C.S., H.C., A.D.J.B., A.M.N.: acquired the data. A.M., J.M., A.M.N., E.K., S.K.P., and C.-P.N.: analyzed the data. A.M., J.M., and A.M.N.: wrote the paper. C.S., H.C., T.F., J.B., A.D.J.B., and K.M.: critically revised the manuscript. A.M., C.S., T.F., and J.M.: supervised the study.

\section{References}

1 Pokorny MR, de Rooij M, Duncan E, Schröder FH, Parkinson R, Barentsz JO, et al. Prospective study of diagnostic accuracy comparing prostate cancer detection by transrectal ultrasound-guided biopsy versus magnetic resonance (MR) imaging with subsequent MRguided biopsy in men without previous prostate biopsies. Eur Urol. 2014 Jul;66(1):22-9.

2 Siddiqui MM, Rais-Bahrami S, Turkbey B, George AK, Rothwax J, Shakir N, et al. Comparison of MR/ultrasound fusion-guided biopsy with ultrasound-guided biopsy for the diagnosis of prostate cancer. JAMA. 2015 Jan; 313(4):390-7.

3 Barentsz JO, Richenberg J, Clements R, Choyke P, Verma S, Villeirs G, et al.; European Society of Urogenital Radiology. ESUR prostate MR guidelines 2012. Eur Radiol. 2012 Apr;22(4):746-57.

4 Cash H, Maxeiner A, Stephan C, Fischer T, Durmus T, Holzmann J, et al. The detection of significant prostate cancer is correlated with the Prostate Imaging Reporting and Data System (PI-RADS) in MRI/transrectal ultrasound fusion biopsy. World J Urol. 2016 Apr;34(4):525-32.

5 Ahmed HU. The index lesion and the origin of prostate cancer. N Engl J Med. 2009 Oct; 361(17):1704-6.

6 Filson CP, Natarajan S, Margolis DJ, Huang J, Lieu P, Dorey FJ, et al. Prostate cancer detection with magnetic resonance-ultrasound fusion biopsy: the role of systematic and targeted biopsies. Cancer. 2016 Mar;122(6):884-92.

7 Turkbey B, Pinto PA, Mani H, Bernardo M, Pang Y, McKinney YL, et al. Prostate cancer: value of multiparametric MR imaging at $3 \mathrm{~T}$ for detection-histopathologic correlation. Radiology. 2010 Apr;255(1):89-99.

8 Radtke JP, Schwab C, Wolf MB, Freitag MT, Alt CD, Kesch C, et al. Multiparametric Magnetic Resonance Imaging (MRI) and MRITransrectal Ultrasound Fusion Biopsy for Index Tumor Detection: Correlation with Radical Prostatectomy Specimen. Eur Urol. 2016 Nov;70(5):846-53.

9 Hanna N, Wszolek MF, Mojtahed A, Nicaise E, Wu B, Gelpi-Hammerschmidt FJ, et al.
Multiparametric Magnetic Resonance Imaging-Ultrasound Fusion Biopsy Improves But Does Not Replace Standard Template Biopsy for the Detection of Prostate Cancer. J Urol. 2019, Epub ahead of print.

10 Le JD, Tan N, Shkolyar E, Lu DY, Kwan L, Marks LS, et al. Multifocality and prostate cancer detection by multiparametric magnetic resonance imaging: correlation with wholemount histopathology. Eur Urol. 2015 Mar; 67(3):569-76.

11 Kasivisvanathan V, Rannikko AS, Borghi M, Panebianco V, Mynderse LA, Vaarala MH, et al.; PRECISION Study Group Collaborators. MRI-Targeted or Standard Biopsy for Prostate-Cancer Diagnosis. N Engl J Med. 2018 May;378(19):1767-77.

12 Ahmed HU, El-Shater Bosaily A, Brown LC, Gabe R, Kaplan R, Parmar MK, et al.; PROMIS study group. Diagnostic accuracy of multi-parametric MRI and TRUS biopsy in prostate cancer (PROMIS): a paired validating confirmatory study. Lancet. $2017 \mathrm{Feb}$; 389(10071):815-22. 
13 van der Leest M, Cornel E, Israel B, Hendriks R, Padhani AR, Hoogenboom M, et al. Headto-head Comparison of Transrectal Ultrasound-guided Prostate Biopsy Versus Multiparametric Prostate Resonance Imaging with Subsequent Magnetic Resonance-guided Biopsy in Biopsy-naive Men with Elevated Prostate-specific Antigen: A Large Prospective Multicenter Clinical Study. Eur Urol. 2019 Apr;75(4):570-8.

14 Mottet N, Bellmunt J, Bolla M, Briers E, Cumberbatch MG, De Santis M, et al. EAU-ESTRO-SIOG Guidelines on Prostate Cancer. Part 1: Screening, Diagnosis, and Local Treatment with Curative Intent. Eur Urol. 2017 Apr;71(4):618-29.

15 Djavan B, Ravery V, Zlotta A, Dobronski P, Dobrovits M, Fakhari M, et al. Prospective evaluation of prostate cancer detected on biopsies 1, 2, 3 and 4: when should we stop? J Urol. 2001 Nov;166(5):1679-83.

16 Ploussard G, Nicolaiew N, Marchand C, Terry S, Allory Y, Vacherot F, et al. Risk of repeat biopsy and prostate cancer detection after an initial extended negative biopsy: longitudinal follow-up from a prospective trial. BJU Int. 2013 May;111(6):988-96.

17 Haffner J, Potiron E, Bouyé S, Puech P, Leroy $\mathrm{X}$, Lemaitre L, et al. Peripheral zone prostate cancers: location and intraprostatic patterns of spread at histopathology. Prostate. 2009 Feb;69(3):276-82.

18 Mygatt J, Sesterhenn I, Rosner I, Chen Y, Cullen J, Morris-Gore T, et al. Anterior tumors of the prostate: clinicopathological features and outcomes. Prostate Cancer Prostatic Dis. 2014 Mar;17(1):75-80.

19 Boesen L, Nørgaard N, Løgager V, Balslev I, Thomsen HS. Where Do Transrectal Ultrasound- and Magnetic Resonance Imagingguided Biopsies Miss Significant Prostate Cancer? Urology. 2017 Dec;110:154-60.

20 Krughoff K, Eid K, Phillips J, Stoimenova D, Smith D, O'Donnell C, et al. The accuracy of prostate cancer localization diagnosed on transrectal ultrasound-guided biopsy compared to 3-dimensional transperineal approach. Adv Urol. 2013;2013:249080.
21 Schouten MG, Hoeks CM, Bomers JG, Hulsbergen-van de Kaa CA, Witjes JA, Thompson LC, et al. Location of Prostate Cancers Determined by Multiparametric and MRI-Guided Biopsy in Patients With Elevated ProstateSpecific Antigen Level and at Least One Negative Transrectal Ultrasound-Guided Biopsy. AJR Am J Roentgenol. 2015 Jul;205(1):57-63.

22 Wegelin O, van Melick HH, Hooft L, Bosch JL, Reitsma HB, Barentsz JO, et al. Comparing Three Different Techniques for Magnetic Resonance Imaging-targeted Prostate Biopsies: A Systematic Review of In-bore versus Magnetic Resonance Imaging-transrectal Ultrasound fusion versus Cognitive Registration. Is There a Preferred Technique? Eur Urol. 2017 Apr;71(4):517-31.

23 Moussa AS, Meshref A, Schoenfield L, Masoud A, Abdel-Rahman S, Li J, et al. Importance of additional "extreme" anterior apical needle biopsies in the initial detection of prostate cancer. Urology. 2010 May;75(5): 1034-9.

24 O'Neil LM, Walsh S, Cohen RJ, Lee S. Prostate carcinoma with positive margins at radical prostatectomy: role of tumour zonal origin in biochemical recurrence. BJU Int. 2015 Oct; 116 Suppl 3:42-8.

25 Dickinson L, Ahmed HU, Allen C, Barentsz JO, Carey B, Futterer JJ, et al. Magnetic resonance imaging for the detection, localisation, and characterisation of prostate cancer: recommendations from a European consensus meeting. Eur Urol. 2011 Apr;59(4):477-94.

26 Borkowetz A, Hadaschik B, Platzek I, Toma M, Torsev G, Renner T, et al. Prospective comparison of transperineal magnetic resonance imaging/ultrasonography fusion biopsy and transrectal systematic biopsy in biopsy-naïve patients. BJU Int. 2018 Jan;121(1): 53-60.

27 Cash H, Günzel K, Maxeiner A, Stephan C, Fischer T, Durmus T, et al. Prostate cancer detection on transrectal ultrasonographyguided random biopsy despite negative realtime magnetic resonance imaging/ultrasonography fusion-guided targeted biopsy: reasons for targeted biopsy failure. BJU Int. 2016 Jul;118(1):35-43.
28 Westhoff N, Baeßler B, von Hardenberg J, Hetjens S, Porubsky S, Siegel F, et al. Systematic prostate biopsy still matters: A comprehensive analysis of MRI/TRUS-fusion targeted prostate biopsies across different indications. Urol Oncol. 2019 Oct;37(10):678-87.

29 Abraham NE, Mendhiratta N, Taneja SS. Patterns of repeat prostate biopsy in contemporary clinical practice. J Urol. 2015 Apr;193(4): 1178-84.

30 Leyh-Bannurah SR, Kachanov M, Beyersdorff D, Preisser F, Tilki D, Fisch M, et al. Anterior Localization of Prostate Cancer Suspicious Lesions in 1,161 Patients Undergoing Magnetic Resonance Imaging/Ultrasound Fusion Guided Targeted Biopsies. J Urol. 2018 Nov; 200(5):1035-40.

31 Schouten MG, van der Leest M, Pokorny M, Hoogenboom M, Barentsz JO, Thompson LC, et al. Why and Where do We Miss Significant Prostate Cancer with Multi-parametric Magnetic Resonance Imaging followed by Magnetic Resonance-guided and Transrectal Ultrasound-guided Biopsy in Biopsy-naïve Men? Eur Urol. 2017 Jun;71(6):896-903.

32 Pietzak EJ, Resnick MJ, Mucksavage P, Van Arsdalen K, Wein AJ, Malkowicz SB, et al. Multiple repeat prostate biopsies and the detection of clinically insignificant cancer in men with large prostates. Urology. 2014 Aug; 84(2):380-5.

33 Bittner N, Merrick GS, Butler WM, Bennett A, Galbreath RW. Incidence and pathological features of prostate cancer detected on transperineal template guided mapping biopsy after negative transrectal ultrasound guided biopsy. J Urol. 2013 Aug;190(2):509-14.

34 Gershman B, Zietman AL, Feldman AS, McDougal WS. Transperineal template-guided prostate biopsy for patients with persistently elevated PSA and multiple prior negative biopsies. Urol Oncol. 2013 Oct;31(7):1093-7.

35 Radtke JP, Boxler S, Kuru TH, Wolf MB, Alt $\mathrm{CD}$, Popeneciu IV, et al. Improved detection of anterior fibromuscular stroma and transition zone prostate cancer using biparametric and multiparametric MRI with MRI-targeted biopsy and MRI-US fusion guidance. Prostate Cancer Prostatic Dis. 2015 Sep;18(3):288-96. 